We present a selection of important methods and areas of methodological development worth watching in the coming years.

\section{> CRISPRs and epigenome editing}

Precise alterations to the epigenome with targeted enzymes.

Since we picked genome engineering as the Method of the Year in 2011, the arrival of the CRISPR (clustered regularly interspaced short palindromic repeats) system has been hailed as a revolution for this field. The guide RNA/Cas9 nuclease complex overcomes some of the limitations of previous tools. Guide RNAs are easy to design and can target the Cas9 protein to almost any desired region in the genome. Cas9, used either as a nuclease or nickase, induces breaks in the DNA that are then exploited for gene knockout, insertion of tags or gene replacement. In fact, Cas9's potential goes beyond DNA cleavage-as a mutant without nuclease activity, it has been used to target proteins with specific functions to distinct genomic loci. It is this role of Cas9 that, in our view, is the most promising characteristic of the system and will allow its expansion from a genome-editing tool to one capable of introducing custom changes in the complex epigenome.

Two research groups recently harnessed the more established TALEs (transcription activator-like effectors) to modify the epigenome. Bernstein and colleagues targeted histone marks in enhancers with a demethylase/deacetylase complex to query their role in transcription (Nat. Biotechnol. 31, 1133-1136, 2013), and Zhang and colleagues used TALEs fused to a light-inducible system to target histonemodifying enzymes to endogenous loci and showed a repression of transcription (Nature 7463, 472-476, 2013). Although these approaches are promising, the targeting of TALEs is more cumbersome and labor intensive than targeting enzymes via the CRISPR/Cas9 system. By fusing

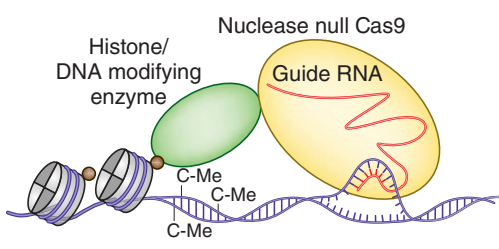

CRISPR/Cas9 modifies the epigenome.

any enzyme of choice to Cas9, one will be able to not only alter histone modification

\section{> Bring on the neuro tools}

A boost to neuroscience technology development could be transformative.

Much excitement has built around neuroscience as the US President announced the launch of the Brain Research through Advancing Innovative Neurotechnologies (BRAIN) initiative in the spring of 2013. This multiyear project will promote technological developments related to basic and applied neuroscience, with the goal of advancing our understanding of how brains compute information and what goes wrong in diseases of the nervous system.

Neuroscientists are bound by their methods and can only 'see' as far as the methods allow them to. Technologies do exist to record the activity of groups of neurons at high spatial and temporal resolution using either imaging approaches or electrodes, and in recent years, we have seen great advances in methods to manipulate this activity using optogenetics. But interesting brain functions likely emerge from the interaction of many interconnected neurons distributed across a large part of the brain. Monitoring and manipulating these large cellular ensembles in the living organism, and pairing their activity to specific behaviors, remains a challenge. Additional developments in optical and non-optical methods will be needed to expand our view and thereby change chromatin states, but also influence DNA methylation, which would allow access to an entirely new repertoire of regulation of cellular function. Whether CRISPRs can indeed be turned into the finely honed scalpels to edit the epigenome without any concerns about off targets will become more obvious in the near future.

Nicole Rusk

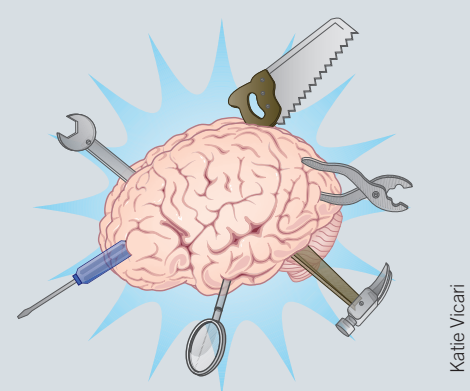

We hope to see important technological developments in neuroscience.

of neuronal activity to include entire interconnected functional circuits.

Of critical importance are biosensors that allow monitoring of and interfering with cellular activity in the living brain. We expect to see major improvements in the performance of genetically encoded voltage sensors and a surge in their in vivo application in the next years. Development of neuronal-activity reporters that are sensitive to the far-red region of the visible light spectrum and increased use of adaptive optics-based methods will be critical to enable deeper in vivo imaging.

We anticipate that technological developments in neuroscience will take many exciting paths in the coming years. A Focus on 'Mapping the Brain' was published in Nature Methods in 2013, http://www.nature. com/nmeth/focus/brainmapping/index. html and we look forward to publishing many methodological developments in this area in the years to come! Erika Pastrana 